\title{
Biometry of the crystalline lens in late onset diabetes: the importance of diabetic type
}

\author{
John M Sparrow, Anthony J Bron, Nicholas A Phelps Brown, H A W Neil
}

\begin{abstract}
Lenticular and anterior chamber biometry were studied in non-cataractous eyes by means of Scheimpflug photography and digital image analysis. The study population consisted of 91 late onset diabetic subjects and 115 nondiabetic controls. Anteroposterior axial lens thickness, cortical thickness, nuclear thickness, anterior clear zone thickness, anterior chamber depth, and anterior and posterior lenticular curvatures were assessed. Age played an important role in determining lens biometry in all subjects, and small but significant differences were found between late onset diabetics and non-diabetics. In the late onset diabetic subgroup, apart from age, diabetic retinopathy was the only significant parameter found which determined lens biometry. These biometric findings in late onset diabetes are in marked contrast to the large overall effect of diabetes and the powerful effect of diabetic duration which we previously reported in early onset diabetes. Further analysis of the data from our previous study has been provided, which clearly demonstrates differences between the impact of early and late onset diabetes on the biometry of the anterior ocular segment.
\end{abstract}

Mitotic activity in the pre-equatorial lenticular epithelium results in steady growth of the human crystalline lens throughout life. ${ }^{1-5}$ Overall lens growth is determined by a dynamic balance between external accretion of secondary lens fibres and the central compaction of older nuclear or perinuclear fibres. ${ }^{36}$ Anteroposterior lens growth is proportionally greater than equatorial growth, anterior and posterior lens curvatures therefore become steeper (shorter radii of curvature) with increasing age. ${ }^{57}$

Lenticular biometry is disturbed in diabetes, ${ }^{5-10}$ with early onset diabetics showing markedly abnormal lens growth with a powerful dependency upon diabetic duration. ${ }^{5}$ The anterior clear zone (first zone of disjunction) of the lens behaves independently of the other lenticular zones. This zone, which is neither age dependent nor dependent upon diabetic duration, has been found to be markedly increased in early onset diabetics. ${ }^{5}$ The increased anterior clear zone thickness and the powerful dependency of other biometric parameters on diabetic duration imply that the lenses of early onset diabetics may not simply be over-hydrated, but that they may be in a state of accelerated growth, with either more (hyperplastic mechanism) or individually larger (hypertrophic mechanism) secondary lens fibres being formed. Intracellular 'swelling' of individual lens fibres would be theoretically possible although such swelling would need to be confined to the newly formed (metabolically active) fibres to be compatible with the powerful duration effect observed in early onset diabetes. ${ }^{5}$ In order for extracellular swelling of the lens to explain the observed effect of duration it would be necessary to postulate that such swelling increased over time with diabetic duration. This is conceivable, as ongoing cumulative damage to membrane structures could take place over periods of years.

Previous studies have not examined specifically the effect of late onset diabetes on lenticular biometry. ${ }^{5910}$ The present study examines biometry in clear lenses of late onset diabetics and controls, and draws comparisons between the impact of diabetes on the lens in late and early onset diabetes.

\section{Material and methods}

\section{SUBJECTS}

A total of 91 late onset diabetics ( 57 males) and 115 non-diabetic controls (64 males) were included in the study. These patients formed part of a population-based comparative lens study. ${ }^{11}$ The diabetics were survivors of the Oxford Community Diabetes Study, ${ }^{12}$ and community-based controls were selected to group match the diabetics by age and sex. In the present study only the late onset diabetics were included. Late onset diabetics were regarded simply as all those diabetics who did not fit the inclusion criteria used in our earlier study for early onset diabetes. In effect this meant that late onset diabetics were defined as any diabetic whose age at onset was more than 30 years regardless of the type of diabetic treatment, or diabetics whose age at onset was 30 years or less, but who did not require continuous insulin treatment. Controls were all community based, and were accepted if they had no history of diabetes or impaired glucose tolerance, and a non-fasting whole venous blood glucose of less than $7.8 \mathrm{mmol} / \mathrm{l} .{ }^{131+}$

Individual eyes of diabetics and controls were included in the present study if the lenses were non-cataractous, and had normal anterior ocular segments and vision sufficiently good to hold fixation during Scheimpflug photography. Cataractous lenses were excluded because such lenses may have abnormal biometry, ${ }^{21516}$ although certain minor opacities as defined by the Oxford Clinical Cataract Classification and Grading System ${ }^{1718}$ were accepted: nuclear brunescence and white nuclear scatter up to and including Grade 2, spoke opacities and waterclefts of Grade 1, and isolated vacuoles, retrodots and focal dots. No anterior or posterior sub- 
capsular opacities were permitted, as subcapsular opacities were specifically known to be associated with reduced lens size. ${ }^{15}$ There were thus a total of 378 eligible eyes in 206 subjects; 188 were right eyes and 190 were left eyes.

The study was approved by the Central Oxford Research Ethics Committee (ref no 1211), and informed consent was obtained from participants.

\section{PROCEDURE}

The procedure followed has been described. ${ }^{5}$ Briefly, subjects were contacted and recruited by the primary investigator (JS). Following a short history and Snellen acuity testing pupils were dilated (tropicamide $1 \%$ and phenylephrine $10 \%$ ) with due regard to the usual precautions. Lenses were assessed for cataract at the slit-lamp biomicroscope, and Scheimpflug photographs were taken using a Brown Scheimpflug camera. ${ }^{19-21}$ Biometric measurements on digitised images were performed by a masked observer (JS) using the Oxford Modular Cataract Image Analysis System which was developed by the authors. ${ }^{22}$ As in our previous study of lens biometry in early onset diabetes ${ }^{5}$ the biometric parameters measured were: anteroposterior axial lens thickness, cortical thickness, nuclear thickness, anterior clear zone thickness, anterior chamber depth, and anterior and posterior lenticular curvatures. Cortical thickness was derived arithmetically as the difference between lens and nuclear thickness.

\section{STATISTICAL METHODS}

Biometric measurements were in general obtained from both eyes, although certain patients contributed measurements on only one eye. A preliminary analysis was performed by plotting the biometric data against age and performing simple linear regression for the diabetic and non-diabetic groups separately. In the main analysis the within-subject intereye correlation was accounted for by employing the intraclass correlation model of Rosner for calculating significance levels and confidence intervals. ${ }^{23}$ The dependent variables were continuous and were approximately normally

Figure 1 Frequency distribution of the ages of late onset diabetics (cases) and non-diabetics (controls).

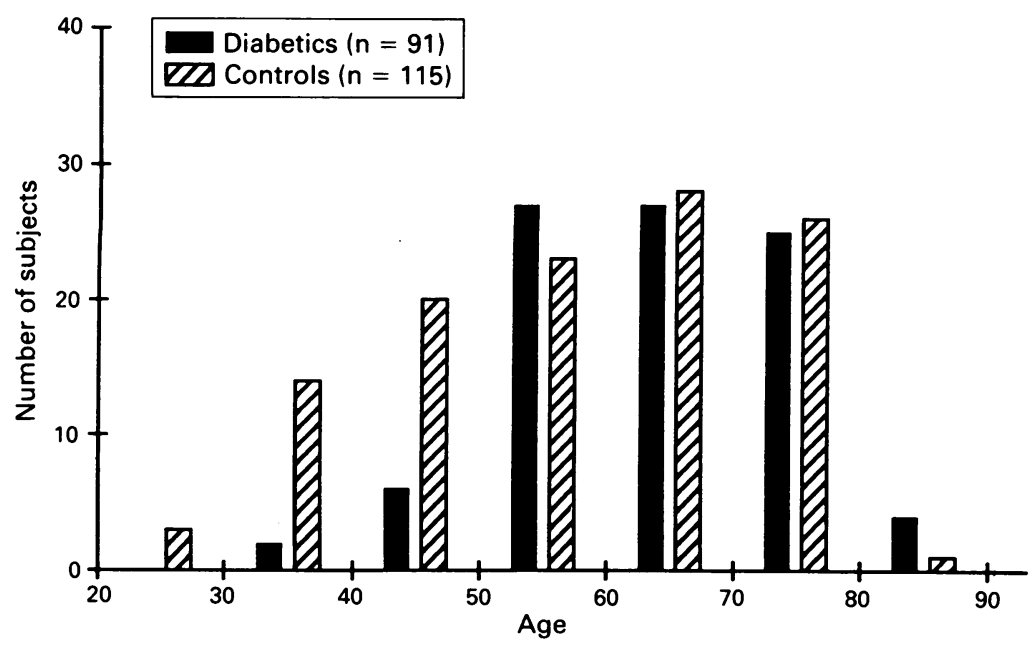

multiple linear regression analysis with groups (factors), the biometric measures being used as dependent variables in a series of model fitting exercises. Adjustment for minor imbalances in the case/control age distribution was achieved by treating age as a covariate in the relevant analyses. To facilitate comparison with our earlier study of lens biometry in early onset diabetes the analyses were performed in broadly the same manner as in that study..$^{5}$ (The orders of the fitting of terms to the models are indicated in the tables.) Using the data from the previous study as well as the data from the present study additional analyses were performed comparing the overall effects of early and late onset diabetes with non-diabetic controls, and comparing early and late onset diabetics directly. These analyses provide the average group differences (with $95 \%$ confidence intervals) between both types of diabetics and controls, and between early and late onset diabetics, after taking account of the (confounding) effects of age and sex. The analysis was performed using the Generalised Linear Interactive Modelling (GLIM) System of the Numerical Algorithms Group (NAG, Wilkinson House, Jordan Hill Road, Oxford OX2 8DR).

\section{Results}

The age distribution of the late onset diabetics and controls is presented in Figure 1. The age/ duration distributions for the diabetics alone are presented in Table 1. Figures 2 to 8 illustrate the preliminary regression analyses of biometry against age. These graphs demonstrate the important effect of age on anterior segment biometry. For certain of the biometric features studied there appear to be small differences between the late onset diabetics and controls.

These impressions were investigated in the formal analysis summarised in Table 2 . The 'late onset diabetics and controls' analysis (206 subjects, 378 eyes) confirmed that age was an important determinant of biometry. Modest differences existed between diabetics and nondiabetics for axial lens thickness, cortical thickness, and front radius of curvature. These changes were such that lenses of diabetics were 'thicker' with steeper front curvatures. The significant age by diabetic status interaction term (age.sta) for front radius of curvature indicated that the younger diabetics were relatively more affected, this being well demonstrated in Figure 7 (regression lines non-parallel). A substantial difference in anterior clear zone thickness between diabetics and non-diabetics was found, with diabetics having increased clear zone thick-

Table 1 Age by diabetic duration of the late onset diabetes study population

\begin{tabular}{lccccc}
\hline \multicolumn{7}{c}{ Diabetic duration (years) } \\
Age (years) & $<10$ & $<20$ & $<30$ & $30+$ & Total \\
\hline 30 to $<40$ & 2 & 0 & 0 & 0 & 2 \\
40 to $<50$ & 4 & 2 & 0 & 0 & 6 \\
50 to $<60$ & 10 & 13 & 4 & 0 & 27 \\
60 to $<70$ & 10 & 14 & 1 & 2 & 27 \\
70 to $<80$ & 6 & 11 & 7 & 1 & 25 \\
$80+$ & 2 & 0 & 2 & 0 & 4 \\
Total & 34 & 40 & 14 & 3 & 91 \\
\hline
\end{tabular}


ness. Female sex was associated with decreased cortical thickness.

In the late onset diabetic subgroup (91 subjects, 161 eyes) age was an important determinant of lens biometry for all parameters except nuclear thickness and anterior clear zone

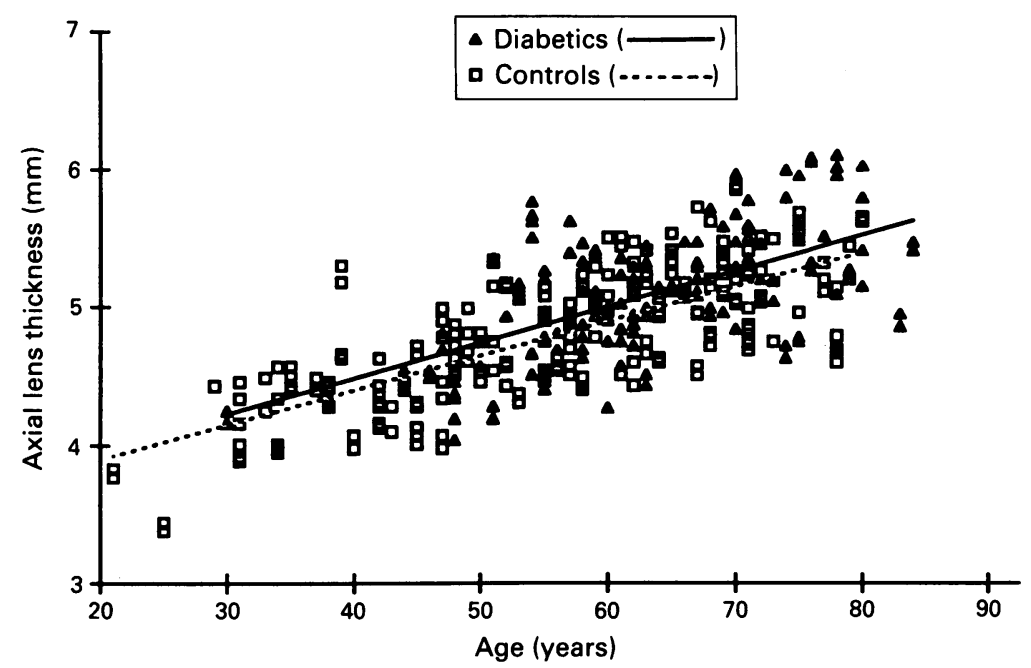

Figure 2 Plot of anteroposterior axial lens thickness derived from image analysis of Scheimpflug photographs against age. Separate linear regression lines for diabetics and controls.

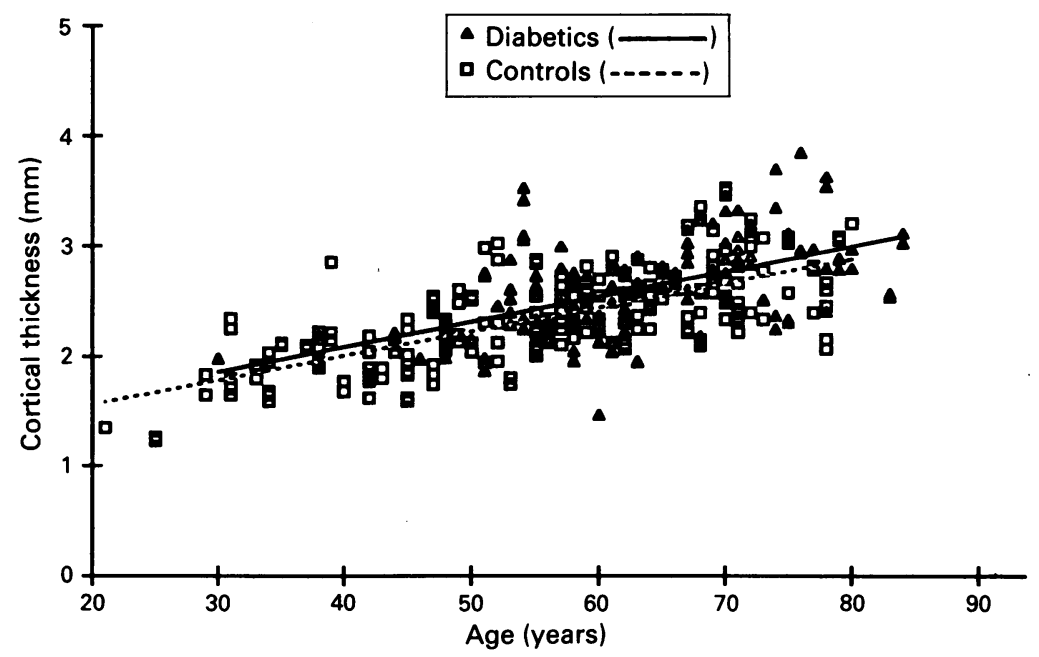

Figure 3 Plot of anteroposterior cortical thickness of the lens derived from image analysis of Scheimpflug photographs against age. Separate linear regression lines for diabetics and controls.

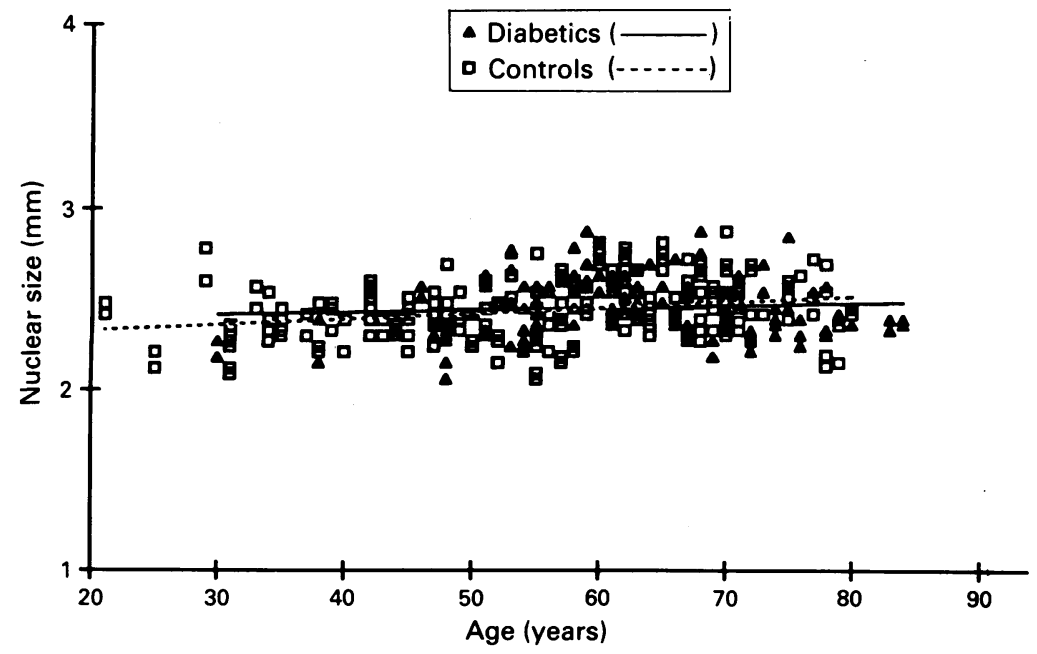

Figure 4 Plot of anteroposterior nuclear size of the lens derived from image analysis of Scheimpflug photographs against age. Separate linear regression lines for diabetics and controls. thickness. Proliferative diabetic retinopathy was associated with increased lens and cortical thickness. Diabetic duration, sex, or type of diabetic treatment (insulin, oral hypoglycaemics, or diet) did not have a demonstrable effect on any of the biometric parameters studied.

Among the controls (115 subjects, 217 eyes) age was an important determinant of biometry, with female sex associated with decreased axial length and cortical thickness and with increased nuclear thickness.

The differences between the late onset diabetics and controls were observed to be less impressive than those previously found for 153 early onset diabetics and 153 controls $^{5}$ (the 115 controls in the present study being a subset of the previous 153). For this reason further analyses making a direct comparison between early and late onset diabetics were performed. The first of these analyses is summarised in Table 3 , and demonstrated important differences between the two groups of diabetics (after accounting for confounding effects). The differences between the early and late onset groups were such that the lenses of the early onset patients were significantly larger in their axial, cortical, and nuclear thicknesses, had steeper front and back radii of curvatures, and were associated with shallower anterior chambers. The interaction terms between age and type of diabetes (age.type) indicated certain differences in the slopes of the regression lines between the two groups. The regression lines for lens thickness (cortical thickness) and front radius of curvature were each less steep in the late onset diabetics than in the early onset diabetics, indicating that the average change per year in biometry was greater in the early onset group.

The magnitudes of the observed effects (with $95 \%$ confidence intervals) are presented in Table 4. These analyses demonstrated the average effects of late onset and early onset diabetes on lens biometry compared with controls, and also compared early and late onset diabetics directly. After adjustment for age and sex, the effect sizes in the early and late onset diabetics compared with controls indicated that the impact of lens. biometry of early onset diabetes was between two and three times that found in late onset diabetes. The final group of analyses in Table 4 provide direct estimates (adjusted for age and sex) for the overall differences between early and late onset diabetics. These estimates show that diabetics demonstrate a particular pattern of disturbance of anterior segment biometry, and that the magnitude of this disturbance is considerably greater among early onset than among late onset diabetics.

Because the early onset diabetics from our previous study ${ }^{5}$ were on average younger than the late onset diabetics in the present study, two further analyses were performed to determine whether the observed differences might be due to differences in the age structures of the two diabetic populations. To examine this point attention was directed to the impact of diabetic duration, which, as has been noted above, was a powerful effect among the early onset diabetics, but which appeared to play no role in the late onset diabetics. Firstly, the early onset group 
was examined to determine whether the powerful duration effect persisted across the entire age range. For this the early onset diabetics (age range 10 to 74 years) were grouped into age quartiles. The duration effect for the biometric

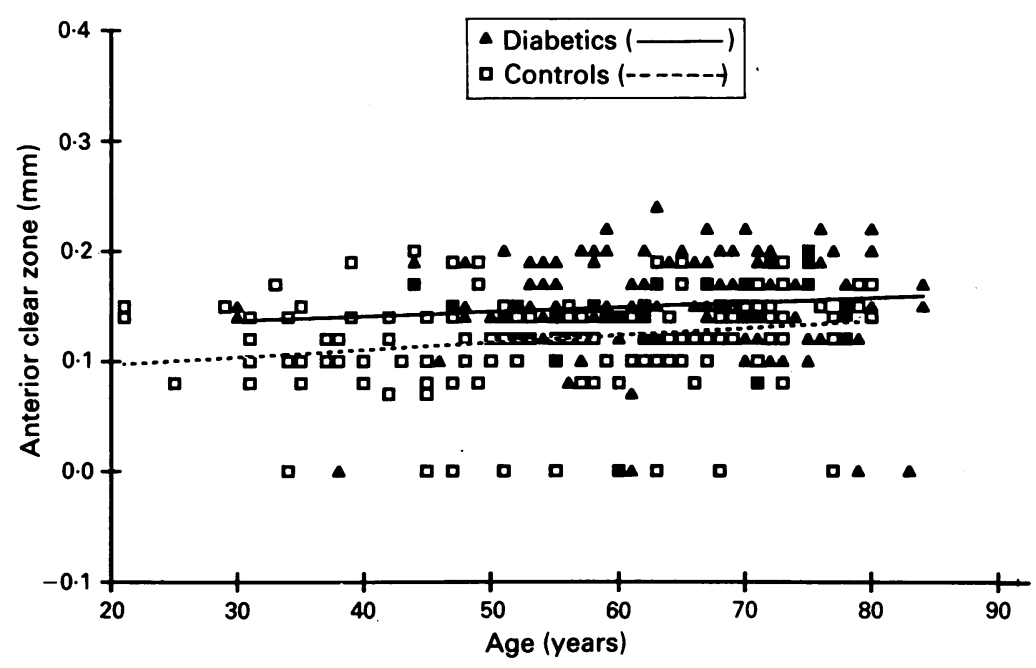

Figure 5 Plot of anterior clear zone thickness of the lens derived from image analysis of Scheimpflug photographs against age. Separate linear regression lines for diabetics and controls.

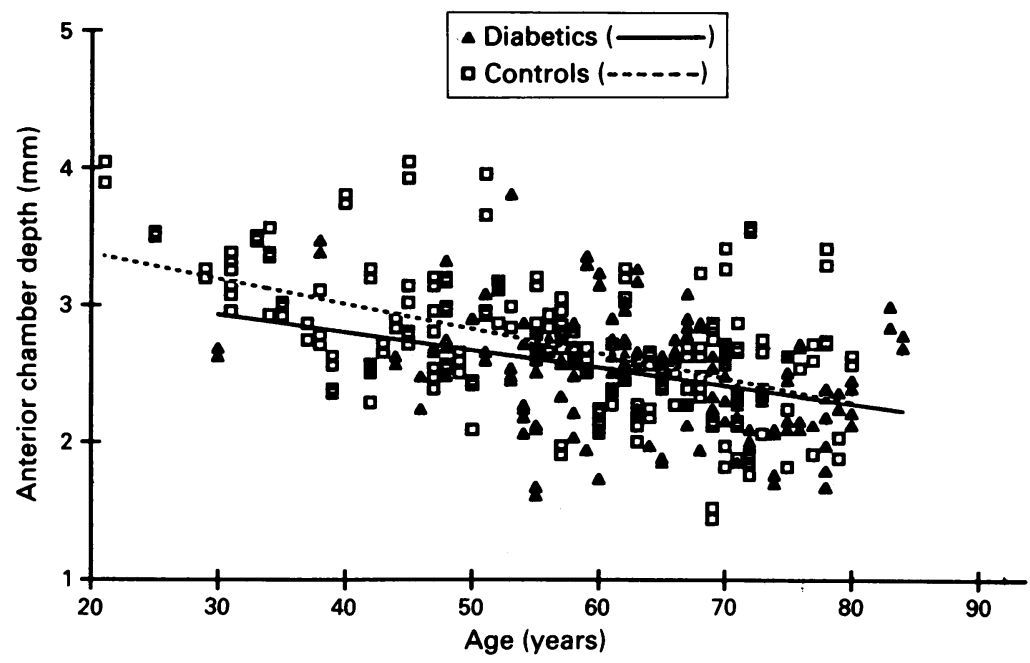

Figure 6 Plot of axial anterior chamber depth of the eye derived from image analysis of Scheimpflug photographs against age. Separate linear regression lines for diabetics and controls.

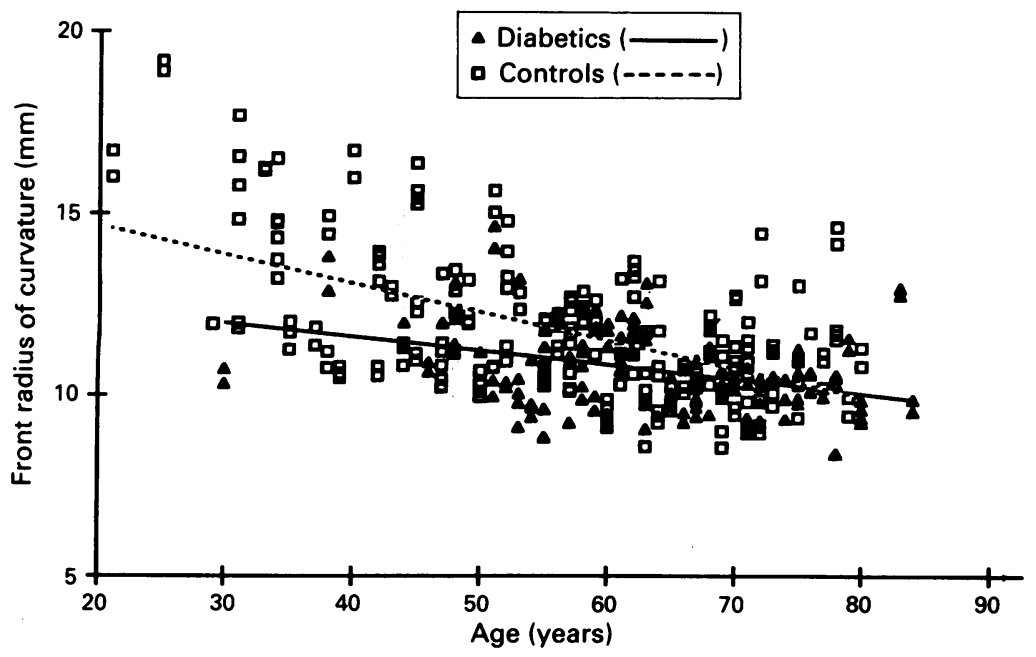

Figure 7 Plot of front radius of curvature of the lens derived from image analysis of Scheimpflug photographs against age. Separate linear regression lines for diabetics and controls. parameters was then determined separately for each age quartile in a model which pre-fixed the age effect to that of the non-diabetic control group, and maintained a constant $y$-intercept for the age quartiles. A summary of this analysis appears in Table 5, which gives the duration estimates (and 95\% confidence intervals) for each quartile. This analysis demonstrated that in general the duration effect among the early onset diabetics persisted across all the age quartiles. In the second analysis all the diabetics (early and late onset) were grouped in deciles according to their age at onset of diabetes. This approach dispensed with the prior classification of the diabetes into early and late onset groups. After adjusting for age and sex each 'onset decile' group was examined separately for an effect of diabetic duration (Table 6). These analyses demonstrated that for most parameters the effect of diabetic duration on biometry diminished dramatically when age at onset of diabetes exceeded 30 years.

\section{Discussion}

The results of this study have demonstrated important differences in the impact of late onset and early onset diabetes on the biometry of the anterior ocular segment. The impact of late onset diabetes on biometry was modest, and a direct comparison between early onset and late onset diabetics confirmed these biometric differences between the two groups of diabetics. Comparisons between each diabetic type and nondiabetic controls revealed that the impact on biometry of early onset diabetes was two to three times greater than the impact of late onset diabetes. Furthermore, within the diabetic subgroups, no effect of diabetic duration was found among the late onset diabetics, which was in marked contrast to the powerful effect of diabetic duration previously found in early onset diabetes. ${ }^{5}$ Further analysis of the data from our earlier study has demonstrated that the duration effect in early onset diabetes persists across all the age quartiles (age range 10 to 74 years). The demonstration that the duration effect persists across the age quartiles is important, because it illustrates that the absence of a duration effect in the late onset diabetics cannot be attributed to the fact that the late onset diabetics were on average older than the early onset diabetics. When the classification of diabetics into early and late onset subjects was dispensed with, age at onset of more than 30 years was noted to be associated with a marked reduction in the effect of diabetic duration after adjustment for the effects of sex and ageing. These findings support the idea that the response of the lens to early onset diabetes is distinct, and is not simply a function of the age of the affected individual. It may be argued that the failure to demonstrate an effect of diabetic duration in the late onset diabetics could be due to the fact that disease duration is frequently not precisely known in such patients. The disease duration of such patients however would in general be underestimated and this would tend to amplify any actual association with duration. Our failure to identify any association with disease duration in 


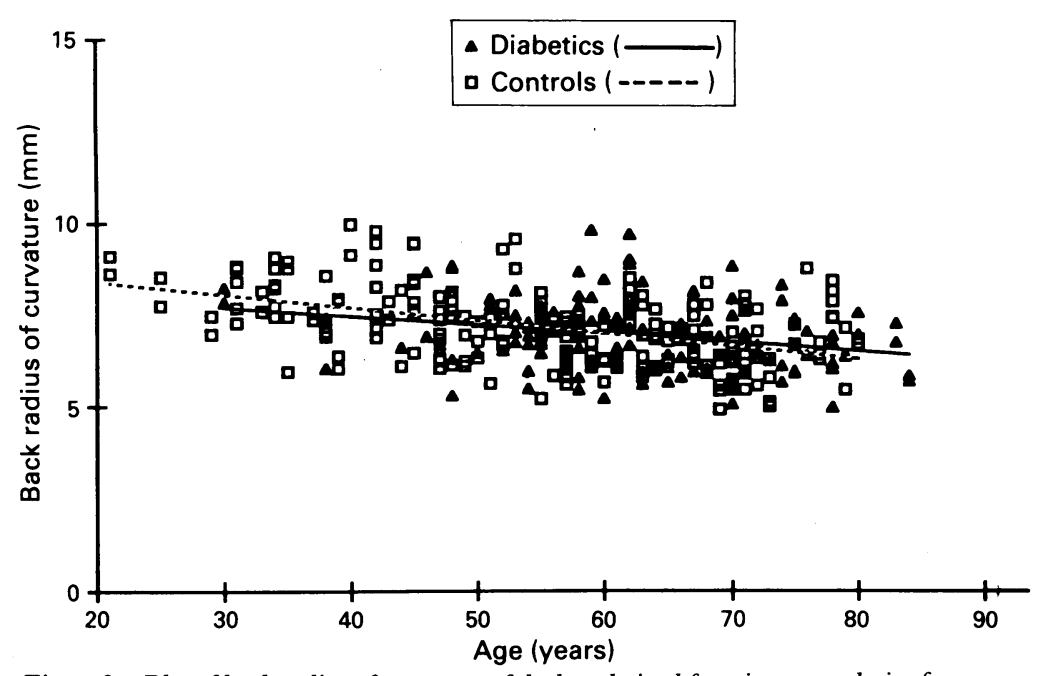

Figure 8 Plot of back radius of curvature of the lens derived from image analysis of Scheimpflug photographs against age. Separate linear regression lines for diabetics and controls.

Table 2 Summary of p values from analysis of lens dimensions in late onset diabetes and controls (clear lenses)

\begin{tabular}{|c|c|c|c|c|c|c|c|}
\hline & Lens & Cortex & Nucleus & $A C Z$ & $A C$ & $F R$ & $B K$ \\
\hline \multicolumn{8}{|c|}{ Late onset diabetics and controls (subjects $=206$, eyes $=378$, right $=188$, left $=190$ ) } \\
\hline Age & $\ll 10^{-6}$ & $\ll 10^{-6}$ & 0.0020 & 0.00031 & $<10^{-6}$ & $<10^{-6}$ & $<10^{-6}$ \\
\hline Sta & 0.029 & 0.034 & NS & 0.000049 & NS & 0.0017 & NS \\
\hline Age.sta & NS & NS & NS & NS & NS & 0.023 & NS \\
\hline Sex & NS & 0.020 & NS & NS & NS & NS & NS \\
\hline $\mathrm{R}$ for model & $0 \cdot 73$ & 0.69 & $0 \cdot 24$ & $0 \cdot 35$ & 0.53 & $0 \cdot 60$ & 0.43 \\
\hline \multicolumn{8}{|c|}{ Late onset diabetes (subjects $=91$, eyes $=161$, right $=81$, left $=80$ ) } \\
\hline Age & $<10^{-6}$ & $<10^{-6}$ & NS & NS & 0.0010 & $0 \cdot 00029$ & 0.0033 \\
\hline Dur & NS & NS & NS & NS & NS & NS & NS \\
\hline Sex & NS & NS & NS & NS & NS & NS & NS \\
\hline Treat & NS & NS & NS & NS & NS & NS & NS \\
\hline BGR & NS & NS & NS & NS & NS & NS & NS \\
\hline PLR & 0.011 & 0.034 & NS & NS & NS & NS & NS \\
\hline $\mathrm{R}$ for model & 0.67 & 0.63 & $0 \cdot 26$ & $0 \cdot 22$ & $0 \cdot 37$ & $0 \cdot 45$ & $0 \cdot 41$ \\
\hline \multicolumn{8}{|c|}{ Controls $($ subjects $=115$, eyes $=217$, right $=107$, left $=110)$} \\
\hline Age & $\ll 10^{-6}$ & $\ll 10^{-6}$ & 0.0024 & 0.014 & $<10^{-6}$ & $<10^{-6}$ & $<10^{-6}$ \\
\hline Sex & 0.024 & 0.00097 & 0.033 & NS & NS & NS & NS \\
\hline $\mathrm{R}$ for model & 0.76 & 0.74 & 0.32 & $0 \cdot 22$ & 0.55 & 0.57 & 0.49 \\
\hline
\end{tabular}

$\mathrm{ACZ}=$ anterior clear zone thickness of lens; $\mathrm{AC}=$ anterior chamber depth; $\mathrm{FR}=$ front radius of curvature of lens; $\mathrm{BK}=$ back radius of curvature of lens; $\mathrm{Sta}=$ diabetic or non-diabetic status; curvature of lens; $\mathrm{BK}=$ back radius of curvature of lens; $\mathrm{Sta}=$ diabetic or non-diabetic sta
Dur $=$ diabetic duration; Treat $=$ diabetic treatment (diet, oral hypoglycaemic or insulin);

Dur=diabetic duration; Treat=diabetic treatment (diet, oral hypoglycaemic or insulin);
$\mathrm{BGR}=$ background retinopathy; $\mathrm{PLR}=$ proliferative retinopathy; $\mathrm{p}$ values calculated by the intraclass correlation model of Rosner. ${ }^{2324}$

Table 3 Summary of $p$ values from analysis of lens dimensions in early and late onset diabetes (clear lenses)

\begin{tabular}{|c|c|c|c|c|c|c|c|}
\hline & Lens & Cortex & Nucleus & $A C Z$ & $A C$ & $F R$ & $B K$ \\
\hline \multicolumn{8}{|c|}{ Early and late onset diabetics $($ subjects $=244$, eyes $=456$, right $=229 ;$ left $=227$ ) } \\
\hline Age & $<<10^{-6}$ & $\ll 10^{-6}$ & 0.00033 & NS & $\ll 10^{-6}$ & $\ll<10^{-6}$ & $<10^{-6}$ \\
\hline Sex & NS & NS & NS & NS & NS & NS & NS \\
\hline Type & $<10^{-6}$ & 0.000001 & 0.00021 & NS & 0.0010 & 0.000033 & 0.00029 \\
\hline Age.type & 0.039 & NS & 0.052 & NS & NS & 0.0021 & \\
\hline $\mathrm{R}$ for model & 0.82 & $0 \cdot 80$ & $0 \cdot 34$ & - & 0.63 & 0.63 & $0 \cdot 37$ \\
\hline
\end{tabular}

$\mathrm{ACZ}=$ anterior clear zone thickness of lens; $\mathrm{AC}=$ anterior chamber depth; $\mathrm{FR}=$ front radius of curvature of lens; $B K=$ back radius of curvature of lens; Type $=$ type of diabetes (early or late onset); p values calculated by the intraclass correlation model of Rosner. ${ }^{2324}$

Table 4 Comparison of the overall effects $( \pm 95 \% \mathrm{CI})$ of diabetes on lens biometry $(\mathrm{mm})$ between controls and diabetics (late and early onset separately), and between early and late onset diabetics, after accounting for the (confounding) effects of age and sex (non-sequential analysis)

\begin{tabular}{|c|c|c|c|c|c|c|c|}
\hline & Lens & Cortex & Nucleus & $A C Z$ & $A C$ & $F R$ & $B K$ \\
\hline $\begin{array}{l}\text { Late onset } \\
\text { vs controls } \\
\quad(n=206)\end{array}$ & $\begin{array}{l}0 \cdot 12^{\star} \\
(0: 093)\end{array}$ & $\begin{array}{l}0 \cdot 11^{\star} \\
(0.091)\end{array}$ & $\begin{array}{l}0.003 \\
(0.042)\end{array}$ & $\begin{array}{l}0 \cdot 026^{\star} \\
(0.012)\end{array}$ & $\begin{array}{l}-0 \cdot 11 \\
(0 \cdot 11)\end{array}$ & $\begin{array}{l}-0 \cdot 71^{\star} \\
(0 \cdot 39)\end{array}$ & $\begin{array}{l}-0 \cdot 022 \\
(0 \cdot 23)\end{array}$ \\
\hline $\begin{array}{l}\text { Early onset } \\
\text { vs controls } \\
(n=306)\end{array}$ & $\begin{array}{l}0.37 \dagger \\
(0.089)\end{array}$ & $\begin{array}{l}0 \cdot 28 \dagger \\
(0.084)\end{array}$ & $\begin{array}{l}0.099^{\star} \\
(0 \cdot 040)\end{array}$ & $\begin{array}{l}0.049 \dagger \\
(0.011)\end{array}$ & $\begin{array}{l}-0.31 \dagger \\
(0 \cdot 10)\end{array}$ & $\begin{array}{l}-1.93 \dagger \\
(0.45)\end{array}$ & $\begin{array}{l}-0.65 \dagger \\
(0.21)\end{array}$ \\
\hline $\begin{array}{l}\text { Early vs } \\
\quad \text { late onset } \\
(n=244)\end{array}$ & $\begin{array}{l}0.43 \dagger \\
(0.14)\end{array}$ & $\begin{array}{l}0 \cdot 31^{\star} \\
(0 \cdot 13)\end{array}$ & $\begin{array}{l}0 \cdot 11^{\star} \\
(0 \cdot 059)\end{array}$ & $\begin{array}{l}0 \cdot 16 \\
(0 \cdot 19)\end{array}$ & $\begin{array}{l}-0 \cdot 25^{\star} \\
(0 \cdot 15)\end{array}$ & $\begin{array}{l}-1 \cdot 32^{\star} \\
(0 \cdot 62)\end{array}$ & $\begin{array}{l}-0 \cdot 62^{\star} \\
(0 \cdot 33)\end{array}$ \\
\hline
\end{tabular}

$\mathrm{ACZ}=$ anterior clear zone thickness of lens; $\mathrm{AC}=$ anterior chamber depth; $\mathrm{FR}=$ front radius of curvature of lens; $\mathrm{BK}=$ back radius of curvature of lens; $95 \% \mathrm{CI}=95 \%$ confidence interval $\star=\mathrm{p}<0.05 ; \mathrm{t}=\mathrm{p}<10^{-6} ;$ confidence intervals and $\mathrm{p}$ values calculated by the intraclass correlation model of Rosner ${ }^{23} 24$; + ve values indicate that the parameter is greater in diabetics $c f$ controls in the first two analyses, and that the parameter is greater in early onset diabetics $c f$ late onset diabetics in the 3rd analysis. late onset patients is therefore unlikely to arise from inaccurate knowledge of true diabetic duration among late onset diabetics. The statistical power of this study to detect a 'medium' sized duration effect among the late onset diabetics in a multiple regression analysis ( $13 \%$ of the variance by Cohen's convention) at the $5 \%$ probability level for 91 subjects is $87 \%$, and for 161 eyes is $99 \% .{ }^{25}$ The true power of the study as analysed by the intraclass correlation model $^{2324}$ will therefore lie between these upper and lower limits, depending on the actual withinsubject intereye correlation for each parameter under study. Even if the intereye correlation was high it would be reasonable to suppose that the power of this study to detect a medium size effect at the $5 \%$ level is at least $90 \%$, which confirms that any duration effect among the late onset diabetics would have had to be small (and therefore of doubtful importance) to have been missed.

Our definitions of early onset diabetes as diabetes diagnosed at or before 30 years of age and requiring continuous insulin treatment, and late onset diabetes as all other diabetics have the effect of separating off a group of young subjects who almost certainly had Type 1 diabetes. This definition of Type 1 diabetes, although based purely on clinical features and age at onset, has been used extensively in epidemiological studies. ${ }^{26}{ }^{27}$ A prospective study of 268 newly diagnosed diabetic patients classified on the basis of clinical criteria only found that all patients diagnosed before the age of 40 years who were clinically assessed as insulin-dependent, had fasting C-peptide levels 18 months after diagnosis which were diagnostic of insulin dependence. ${ }^{28}$ The classification that we used will therefore have ensured that early onset patients were correctly characterised as having minimal endogenous insulin production, although the late onset group is likely to have contained a small number of misclassified patients with true Type 1 (insulin-dependent) diabetes. This might confound comparisons between the early and late onset groups, and it is conceivable (although unlikely) that the 'weak' effects of diabetes seen in our late onset group are (partly) due to the misclassification and inclusion of a small number of truly Type 1 diabetics within a group which should ideally contain only Type 2 patients. Thirty (33\%) of the late onset diabetics were using insulin at the time of the study, $15(16 \%)$ having had their diabetes diagnosed at age $<50$ years, and $10(11 \%)$ at age $<40$ years. The number of potential misclassifications was therefore not very great.

In the present study proliferative diabetic retinopathy was found to be associated with greater lens and cortical thickness, an effect which is in general consistent with our previous finding in early onset diabetics of increased clear zone and nuclear thickness, and steeper front and back curvatures in patients with retinopathy (confounding variables accounted for in each study). These associations raise the possibility that disturbances in lens biometry could be due to the forward diffusion of an (angiogenic) growth factor from the posterior ocular segment, or they may simply imply that patients with a 
Table 5 Slope estimates $( \pm 95 \% \mathrm{CI})$ for the effect of diabetic duration ( $\mathrm{mm} /$ year) on biometry in early onset diabetics across the age quartiles after accounting for the (normal) effect of age

\begin{tabular}{|c|c|c|c|c|c|c|}
\hline Age quartile & $\begin{array}{l}\text { Duration } \\
\text { Lens }\end{array}$ & $\begin{array}{l}\text { ate }(95 \% C I) \\
\text { Cortex }\end{array}$ & Nucleus & $A C$ & $F R$ & $B K$ \\
\hline $1 \mathrm{st}$ & $\begin{array}{l}0.020^{\star} \\
(0.011)\end{array}$ & $\begin{array}{l}0.019^{\star} \\
(0.010)\end{array}$ & $\begin{array}{l}0.0018 \\
(0.0054)\end{array}$ & $\begin{array}{l}-0.023^{\star} \\
(0.013)\end{array}$ & $\begin{array}{l}-0.187 \dagger \\
(0.055)\end{array}$ & $\begin{array}{l}-0.045^{\star} \\
(0.028)\end{array}$ \\
\hline 2nd & $\begin{array}{l}0.019 \dagger \\
(0.007)\end{array}$ & $\begin{array}{l}0.016^{\star} \\
(0.007)\end{array}$ & $\begin{array}{l}0.0033 \\
(0.0034)\end{array}$ & $\begin{array}{l}-0.022 \dagger \\
(0.008)\end{array}$ & $\begin{array}{l}-0.132 \dagger \\
(0.033)\end{array}$ & $\begin{array}{l}-0.030^{\star} \\
(0.017)\end{array}$ \\
\hline 3rd & $\begin{array}{l}0.016 t \\
(0.005)\end{array}$ & $\begin{array}{l}0.013 t \\
(0.005)\end{array}$ & $\begin{array}{l}0.0029^{\star} \\
(0.0025)\end{array}$ & $\begin{array}{l}-0.016 \dagger \\
(0.006)\end{array}$ & $\begin{array}{l}-0.084 \dagger \\
(0.025)\end{array}$ & $\begin{array}{l}-0.016^{\star} \\
(0.013)\end{array}$ \\
\hline 4th & $\begin{array}{l}0.018 t \\
(0.006)\end{array}$ & $\begin{array}{l}0.014 t \\
(0.006)\end{array}$ & $\begin{array}{l}0.0038^{\star} \\
(0.0026)\end{array}$ & $\frac{-0.011^{\star}}{(0.007)}$ & $\begin{array}{l}-0.056^{\star} \\
(0.029)\end{array}$ & $\begin{array}{l}-0.013 \\
(0.017)\end{array}$ \\
\hline
\end{tabular}

$95 \% \mathrm{CI}=95 \%$ confidence interval; $\mathrm{AC}=$ anterior chamber depth; $\mathrm{FR}=$ front radius of curvature of lens; $\mathrm{BK}=$ back radius of curvature of lens; Age quartiles: $1 \mathrm{st}=10$ to $<26 ; 2 \mathrm{nd}=26$ to $<42 ; 3 \mathrm{rd}=42$ to $<58 ; 4$ th $=58$ to 74 years; ${ }^{\star}=p<0 \cdot 05 ; \dagger=p<10^{-6} ;$ confidence intervals and $p$ values calculated by the intraclass correlation model of Rosner. ${ }^{23} 24$

Table 6 Effect of diabetic duration on lens biometry in groups with different age of onset of diabetes after accounting for the (confounding) effects of age and sex

\begin{tabular}{lllllll}
\hline $\begin{array}{l}\text { Age at onset of diabetes } \\
\text { (number of subjects) }\end{array}$ & Lens & Cortex & Nucleus & AC & FR & BK \\
\hline 0 to $<10(43)$ & $<10^{-6 \star}$ & 0.000003 & 0.097 & 0.000016 & $<10^{-6}$ & 0.000008 \\
10 to $<20(60)$ & $<10^{-6}$ & 0.000068 & 0.00021 & 0.0092 & $0 \cdot 000003$ & 0.0036 \\
20 to $<30(43)$ & $0 \cdot 000001$ & $0 \cdot 000004$ & 0.057 & 0.0025 & 0.000013 & 0.039 \\
30 to $<40(19)$ & NS & NS & 0.066 & NS & $0 \cdot 068$ & NS \\
40 to $<50(30)$ & NS & 0.038 & NS & NS & NS & 0.051 \\
50 to $<60(29)$ & NS & NS & NS & NS & NS & NS \\
$60+\quad$ NS & NS & NS & NS & NS & NS \\
\hline
\end{tabular}

$\mathrm{AC}=$ anterior chamber depth; $\mathrm{FR}=$ front radius of curvature of lens; $\mathrm{BK}=$ back radius of curvature of lens; ${ }^{\star} \mathrm{p}$ values calculated by the intraclass correlation model of Rosner. ${ }^{2324}$

'worse' diabetic state tend to be concurrently affected by lens changes and retinopathy. As discussed in our previous publication, 5 disturbance of the actual growth of the lens (by either a hyperplastic or hypertrophic mechanism or both) would be consistent with the finding in early onset diabetes of a powerful diabetic duration effect. With the modest changes found in late onset diabetes, and the absence of any demonstrable effect of diabetic duration, it is necessary to question whether the mechanisms of the disturbances to the lens are different in the two types of diabetes. The argument in favour of a disturbance of actual lens growth in early onset diabetes is partly based upon the finding of a powerful diabetic duration effect in this group, and is perhaps supported indirectly by the observation that biometry is preferentially disturbed in subjects with diabetic retinopathy. In the absence of a duration effect the slightly increased lens thickness in late onset diabetes could be explained simply in terms of an ion/ water imbalance with generalised (extracellular) lens swelling. The idea that lenticular swelling might increase over time (duration) with cumulative membrane damage in early onset diabetes but not in late onset diabetes seems intrinsically unsatisfactory, and argues against 'progressive swelling' of the lens as the mechanism of disturbed biometry in early onset diabetes. Differences between early and late onset diabetics are well recognised, with proliferative diabetic retinopathy. ${ }^{29}$ being commoner, and end stage renal failure ${ }^{1430}$ being about 15 times more common in Type 1 than Type 2 diabetics. Cardiovascular complications on the other hand are commoner among Type 2 diabetics. ${ }^{1431}$

We conclude that in the present study substantial differences in anterior ocular segment biometry have been demonstrated between early and late onset diabetics. These biometric findings may reflect fundamental pathophysiological differences between Type 1 and Type 2 diabetes.

The authors are grateful to Dr D Hockaday, Dr J Mann, Mr H Cheng, Mr P Awdry, and Mr A Freedman for allowing their patients to be recruited to this study, and to Dr J Thompson and Dr J Bithell for statistical advice. This work was supported by Oxford Regional Health Authority NHS Locally Organised Research Grant Number 85/19 and by a British Diabetic Association Research Grant.

1 Weale RA. The aging eye. London: HK Lewis, 1963.

2 Weekers R, Delmarcelle Y, Luyckx-Bacus J, Collignon J. Morphological changes of the lens with age and cataract. In: Elliot $\mathrm{K}$, Fitzsimons DW, eds. The human lens in relation to cataract. CIBA Foundation Symposium 19, 1973; 25-43.

3 Brown NAP. Dating the onset of cataract. Trans Ophthalmol Soc UK 1976; 96: 18-23.

4 Marshall J, Beaconsfield M, Rothery S. The anatomy and development of the human lens and zonules. Trans Ophthalmol Soc UK 1982; 102: 423-40.

5 Sparrow JM, Bron AJ, Brown NAP, Neil HAW. Biometry of the crystalline lens in early-onset diabetes. $\mathrm{Br} \mathcal{F}$ Ophthalmol 1990; 74: 654-60.

6 Brown NAP, Sparrow JM, Bron AJ. Central compaction in the process of lens growth as indicated by lamellar cataract. $\mathrm{Br} f$ Ophthalmol 1988; 72: 538-44.

7 Brown NAP. The change in lens curvature with age. $\operatorname{Exp} E y e$ Res 1974; 19: 175-83.

8 Huggert A. The appearance of the band of disjunction of the lens in diabetes mellitus. Acta Ophthalmol (Kbh) 1953; 31: 227-34.

9 Brown NAP, Hungerford J. The influence of the size of the lens in ocular disease. Trans Ophthalmol Soc UK 1982; 102

10 Fledelius HC, Miyamoto K. Diabetic myopia - is it lensinduced? Acta Ophthalmol (Kbh) 1987; 65: 469-73.

11 Sparrow JM. The lens in diabetes. D Phil Thesis, Linacre College, University of Oxford, 1988.

12 Neil HAW, Gatling W, Mather HM, Thompson AV Thorogood M, Fowler GH, et al. The Oxford community diabetes study: evidence for an increase in the prevalence of known diabetes in Great Britain. Diabet Med 1987; 4: 53943.

13 Diabetes mellitus. Report of WHO study group. WHO technical report series 727 . Geneva: WHO, 1985.

14 Jarrett JR. Diabetes mellitus. (Series in clinical epidemiology). London, Sydney: Croom Helm, 1986.

15 Brown NAP, Tripathy $R$. The loss of the anterior clear zone of the lens, prognostic significance in cataract formation. Tran Ophthalmol Soc UK 1974; 94: 29-45.

16 Perkins ES. Lens thickness in early cataract. $\mathrm{Br} \mathcal{F}$ Ophthalmol 1988; 72: 348-53.

17 Sparrow JM, Bron AJ, Brown NAP, Ayliffe W, Hill AR. The Oxford clinical cataract classification and grading system. Int Ophthalmol 1986; 9: 207-25.

18 Sparrow JM, Ayliffe W, Bron AJ, Brown NAP, Hill AR. Inter-observer and intra-observer variability of the Oxford clinical cataract classification and grading system. Int Ophthalmol 1988; 11: 151-7.

19 Brown NAP. Slit-image photography. Trans Ophthalmol Soc UK 1969; 89: 397-408.

20 Brown NAP. An advanced slit image camera. Br $\mathcal{F}$ Ophthalmol 1972; 56: 624-31.

21 Brown NAP. Quantitative slit-image photography of the lens. Trans Ophthalmol Soc UK 1972; 92: 303-17.

22 Sparrow JM, Brown NAP, Shun-Shin GA, Bron AJ. The Oxford modular cataract image analysis system. Eye 1990; 4 638-48.

23 Rosner B. Statistical methods in ophthalmology: an adjustmen for the intraclass correlation between eyes. Biometrics 1982 38: 105-14.

24 Rosner B. Multivariate methods in ophthalmology with applications to other paired data situations. Biometrics 1984; 40: 1025-35.

25 Cohen J. Statistical power analysis for the behavioral sciences. Chapter 9, 2nd ed. London: Lawrence Erlbaum, 1988

26 Green A, Haughe M, Holm NN, Rasch LL. Epidemiological studies of diabetes mellitus in Denmark. II. A prevalence study based on insulin prescriptions. Diabetologica 1981; 20 468-70.

27 Borch-Johnson K, Kreiner S, Deckert T. Mortality of Type (insulin-dependent) diabetes in Denmark. Diabetologica 1986; 29: 767-72

28 Agner T, Danem P, Binder C. Remission in insulin-dependent diabetes mellitus: a prospective study of basal C-peptide and insulin dose in 286 consecutive patients. Diabetes Care 1987 10: 164-9.

29 Klein R, Klein BEK. Vision disorders in diabetes. In: Diabetes in America. Chapter XIII. US Department of Health \& in America. Chapter XIII. US Department of Heal

30 Herman WH, Teutsch SM. Kidney disease associated with diabetes mellitus. In: Diabetes in America. Chapter XIV. US diabetes mellitus. In: Diabetes in America. Chapter XIV. US no 85-1468, 1985.

31 Pyorala K, Laakso M. Macrovascular disease in diabetes mellitus. In: Mann JI, Pyorala K, Teuscher A, eds. Diabetes in epidemiological perspective. Edinburgh: Churchill Living stone, $1983 ; 7-42$. 